\section{Severe ED causes greater psychosocial impairment than previously thought}

Erectile dysfunction (ED) is associated with psychological dysfunction, but this finding is based on comparisons of men with and without ED. Latini and colleagues, therefore, explored the relationship between ED and psychosocial impairment; they found that as the severity of ED worsens, psychological dysfunction also worsens.

The authors assessed baseline clinical data and responses to a questionnaire from 162 men with ED who participated in the Exploratory and Comprehensive Evaluation of ED study. The cohort was predominantly white (86\%), well-educated (48\% had a college degree) and married (74\%); many (44\%) were employed full-time. In total, 27 men had mild ED, 41 had moderate ED and 94 had severe ED. The questionnaire assessed multiple, validated, ED-specific measures of health-related quality of life, and measures of psychological functioning. Interestingly, patients' and physicians' assessments of ED severity differed; Latini et al. suggest that patients might (perhaps unconsciously) over-represent the severity of their ED, or that their assessment of its severity might be influenced by the amount of psychological distress suffered.

Men with severe ED reported the most depression, anxiety and loneliness, although these impairments were generally insufficient to require psychotherapy. Low sexual selfefficacy scores correlated closely with severe ED; Latini et al. infer that patients' confidence in their sexual abilities is strongly linked to the amount of ED-related psychological distress they experience. These authors call for a multidisciplinary approach to treatment for men with severe ED, and recommend increased use of psychosocial interventions.

Original article Latini DM et al. (2006) Clinical and psychosocial characteristics of men with erectile dysfunction: baseline data from ExCEED ${ }^{\mathrm{TM}}$. J Sex Med 3: 1059-1067

\section{Adjuvant ADT might not improve survival for men with low-risk prostate cancer}

Randomized, clinical trials have demonstrated a clear survival benefit for men with high-risk (locally advanced) prostate cancer who receive advujant androgen-deprivation therapy (ADT) in addition to radiotherapy, and these findings have led to widespread use of this treatment-even for men with low-risk (organ-confined) tumors. Zeliadt and colleagues assessed prostate-cancer survival in a large, US-population-based cohort of men treated with adjuvant ADT and radiotherapy during the 1990s; they found the first population-level evidence of a survival benefit for this therapy only in men with high-risk disease.

Data for the study were obtained from the Surveillance, Epidemiology, and End Results and Medicare databases. The authors identified 31,643 men aged 65-85 years with nonmetastatic prostate cancer who had received adjuvant ADT in combination with radiotherapy. Men with T3-T4 prostate cancer experienced improved 5-year and 8-year survival, but those with stage T1-T2 prostate cancer showed no survival benefit over the 8 years following therapy, although it is possible that a benefit might be observed with extended follow-up. Instrumental variable analysis, a statistical tool that replicates randomization in observational data, was used to control for unmeasured selection bias.

ADT, especially long-term ADT, is associated with considerable side effects and morbidity, which should be weighed against its potential survival benefit. The authors advise that clinicians should follow current practice guidelines, which recommend that adjuvant ADT should be added to radiotherapy only for men with high-risk prostate cancer.

Original article Zeliadt SB et al. (2006) Survival benefit associated with adjuvant androgen deprivation therapy combined with radiotherapy for high- and low-risk patients with nonmetastatic prostate cancer. Int J Radiat Oncol Biol Phys 66: 395-402

\section{Successful neuromodulation might improve sexual function as well as LUTS}

Lower urinary tract dysfunction profoundly affects quality of life in both male and female patients, but their sexual functioning is rarely assessed. Van Balken and colleagues, therefore, assessed the prevalence of sexual dysfunction in a Dutch cohort of patients with overactive bladder, chronic pelvic pain, or nonobstructive urinary retention. The authors found that patients 